\title{
Kernos
}

Revue internationale et pluridisciplinaire de religion grecque antique

$25 \mid 2012$

Varia

\section{Onofrio vox (éd.), Materiali di nomenclatura divina greca}

\section{Sylvain Lebreton}

\section{(2) OpenEdition \\ Journals}

\section{Édition électronique}

URL : http://journals.openedition.org/kernos/2062

DOI : 10.4000/kernos.2062

ISSN : 2034-7871

\section{Éditeur}

Centre international d'étude de la religion grecque antique

\section{Édition imprimée}

Date de publication : 26 octobre 2012

Pagination : 358-360

ISSN : 0776-3824

\section{Référence électronique}

Sylvain Lebreton, «Onofrio vox (éd.), Materiali di nomenclatura divina greca », Kernos [En ligne], 25 |

2012, mis en ligne le 01 octobre 2012, consulté le 21 septembre 2020. URL : http://

journals.openedition.org/kernos/2062 ; DOI : https://doi.org/10.4000/kernos.2062

Ce document a été généré automatiquement le 21 septembre 2020.

Kernos 


\title{
Onofrio vox (éd.), Materiali di nomenclatura divina greca
}

\author{
Sylvain Lebreton
}

\section{RÉFÉRENCE}

Onofrio vox (a cura di), Materiali di nomenclatura divina greca, Lecce, Pensa Multimedia, 2008. 1 vol. $14 \times 20,5$ cm, 130 p. (Quaderni Satura). ISBN : 978-88-8232-653-1.

1 Si les différentes formes de dénomination du divin dans l'antiquité grecque ont depuis longtemps retenu l'attention des historiens et des philologues, elles constituent sans doute l'objet d'étude spécifique d'un nombre croissant de travaux depuis une quinzaine d'années. C'est dans cette optique que s'inscrit ce petit ouvrage collectif regroupant quatre études consacrées aux « modi di denominare, predicare le polimorfiche divinità nella civiltà letteraria greca», sous la direction d'Onofrio Vox. Dans une courte introduction (Premessa, p.5-7), ce dernier passe en revue, dans un continuum assez curieux, les différentes études sur le sujet depuis Socrate de Cos jusqu'aux Götternamen de H. Usener; il propose ensuite une sélection d'instruments plus récents utiles à la recherche dans le domaine, complétée à la fin de chaque chapitre par des indications bibliographiques spécifiques à chacune des quatre contributions.

Dans la première, Mario Andreassi (Il ritratto di Eros in Meleagro AP 5.177, p. 9-37) propose un commentaire de l'épigramme 177 du livre $\mathrm{V}$ de l'Anthologie Palatine, dans laquelle Méléagre de Gadara exploite le thème de l'Éros fugitif au moyen de nombreuses épithètes. L'analyse serrée et rigoureuse porte ainsi attention à chacune d'entre elles, mais aussi à la composition d'ensemble: ainsi toxotès, qui ouvre le dernier vers du poème, n'évoquerait pas tant l'image courante de l'Éros archer qu'un vers de l'Iliade (XI 385). Surtout, ce qualificatif permet à Méléagre de susciter un véritable «colpo di scena ", selon l'A., puisqu'il fait finalement d'Éros, d'abord traqué comme un esclave fugitif, un chasseur qui tient en joue le poète, embusqué dans les yeux de celle qu'il courtise. Ainsi, à travers ce commentaire linéaire, c'est bien la cohérence et l'habileté 
de la construction de l'épigramme qui sont mises en lumière: si la succession d'épithètes permet bien de brosser un portrait d'Éros tout en ambiguïté, elle participe surtout de la dynamique d'ensemble du poème.

3 C'est ensuite une sélection de listes d'épithètes divines que nous propose Claudio Rosato (Liste di epiteti divini nei testi letterari greci e latini. Una raccolta, p. 39-88), non sans en avoir d'abord défini les critères d'établissement grammaticaux: ont ainsi été retenues les listes (définies comme telles à partir de trois épithètes) au sens strict, mais aussi des textes d'autre nature dont l'ossature en est visiblement une. Le recueil fait ainsi la part belle aux scholiastes et compilateurs et - c'est un des principaux mérites de l'entreprise - met à disposition du lecteur des sources peu connues ou peu accessibles (Longibardos ${ }^{1}$ e.g.). Toutefois, si les textes sont présentés avec toute la rigueur philologique attendue, leur classement ne rend pas la consultation toujours aisée (la figuration des épithètes en caractères gras facilite cependant la lecture) : une numérotation aurait ainsi pu être utile et un classement autre que celui adopté (par ordre alphabétique des noms d'auteurs), chronologique ou thématique par exemple, aurait sans doute été plus pertinent. Surtout, on reste frustré par ce qui n'est qu'une récolte : si l'A. précise bien que son recueil ne saurait être exhaustif, ce que l'on ne peut lui reprocher dans le cadre qui lui est ici imparti, le manque de clarté dans les critères motivant la sélection des textes nuit à la cohérence de l'entreprise. Un bornage plus clair du champ (stylistique, chronologique...) dans lequel l'A. a glané ses textes, notamment, aurait ainsi permis au lecteur de mieux prendre la mesure de l'intérêt documentaire de sa récolte.

4 Les deux dernières contributions portent sur les épithètes divines utilisées au pluriel, respectivement chez Eschyle et chez Pollux. Andrea Toma (Epiteti di collettività divine nelle tragedie di Eschilo, p. 89-104) souligne d'emblée la dimension essentiellement politique des divinités invoquées collectivement chez le tragique. Il propose ensuite un commentaire de chacune des épithètes, en mobilisant des parallèles (littéraires pour l'essentiel) afin d'en éclairer la signification, i.e. de montrer la cohérence de leur utilisation avec les préoccupations des personnages qui les invoquent, mais sans toujours les relier avec leur possible contexte cultuel.

Dans une démarche similaire, Onofrio Vox (Nomenclatura divina in Giulio Polluce, p. 105-130) insiste sur l'importance de la nomenclature divine chez Julius Pollux, que traduit peut-être d'emblée le fait que le grammairien entame son Onomasticon par le vocabulaire religieux. En témoigne notamment la longue liste d'épithètes divines que l'on trouve en I, 23-24, dont chacune des composantes est ici classée par champs lexicaux et commentée. Surtout, l'A. questionne l'économie globale de la liste : ainsi, le fait que celle-ci s'ouvre par les appellations relatives aux domaines physiques de résidence et de compétence (ciel et éther; terre et mer; enfers) s'inscrit certes dans des conceptions religieuses "traditionnelles", mais aussi et surtout bien contemporaines de Pollux, trahissant probablement une influence médio-platonicienne. Autre élément notable, la mention de la totalité de la liste au pluriel (quand certaines épithètes sont clairement identifiables comme l'apanage d'un dieu en particulier, Zeus en tête) appelait également un commentaire: l'A.y voit l'expression d'une mentalité syncrétique tolérante, le rhéteur se faisant ici peut-être l'interprète de l'idéologie impériale de Commode ${ }^{2}$. On salue cet effort pour situer Pollux dans son contexte religieux, même si l'on pouvait espérer une interprétation un peu plus approfondie de l'ensemble des données; sans nécessairement y apporter de réponse définitive, il aurait 
été ainsi légitime de tenter de déterminer ce que cette multiplication du pluriel peut nous dire du polythéisme au $\mathrm{II}^{\mathrm{e}}$ siècle de notre ère.

6 Et c'est là un reproche que l'on peut faire à l'ensemble de l'ouvrage : l'historien de la religion grecque antique risque de rester quelque peu frustré à sa lecture, attendant en vain que ces dénominations divines, si savamment présentées et commentées, soient exploitées dans l'optique d'une meilleure lecture du polythéisme hellénique, si ce n'est dans son ensemble, du moins à travers certaines de ses figures. On ne peut certes pas attendre de ces contributions, qui visent avant tout à fournir du « matériau » sur les formes de dénomination divine, ce qu'elles ne nous promettent pas. On regrette cependant que la démarche ne soit pas assez synthétique ou systémique, i.e. envisageant les épithètes non pas séparément, mais dans des ensembles, regret d'autant plus aigu que les sujets et les sources retenus allaient dans ce sens. La faute, peut-être, à l'absence d'une vraie définition du sujet (ce que les A. entendent par "nomenclature » n'est jamais précisément et explicitement défini; de même, les différences entre épithètes poétiques, épiclèses, etc. ne sont pas remises à plat) et d'une problématique générale qui aurait donné à l'ensemble plus de cohérence et de relief³

\section{NOTES}

1. On peut renvoyer le lecteur à l'édition critique de l'œuvre de ce grammairien byzantin (probablement actif au $\mathrm{X}^{\mathrm{e}}$ ou XI ${ }^{\mathrm{e}}$ siècle) proposée par N. FESTA, «Longibardos », Byzantion 6 (1931), p. 101-222.

2. Vox suit ici G. ZECCHINI, «Polluce e la politica culturale di Commodo », in C. BEARZOT, F. LANDUCCI, G. ZECCHINI (éds), L'Onomasticon di Giulio Polluce. Tra lessicografia e antiquaria, Milano, 2007, p. 17-26.

3. L'absence de référence à des travaux qui posent précisément de telles questions, tels que $P$. BRULÉ, « Le langage des épiclèses dans le polythéisme hellénique », Kernos 11 (1998), p. 13-34 ou R. PARKER, "The problem of the greek cult epithet », OAth 28 (2003), p. 173-183, est à cet égard symptomatique.

\section{AUTEURS}

\section{SYLVAIN LEBRETON}

Université de Rennes 2 